\title{
When more is less: Nonmonotonic trends in adsorption on clusters in alloy surfaces
}

\author{
Abigale P. Monasterial, ${ }^{1,2}$ Calla A. Hinderks, ${ }^{1,3}$ Songkun Viriyavaree, ${ }^{1}$ Matthew M. Montemore ${ }^{1, *}$ \\ ${ }^{1}$ Department of Chemical and Biomolecular Engineering, Tulane University, New Orleans, LA \\ 70118, USA \\ ${ }^{2}$ Department of Chemical and Biomolecular Engineering, University of Connecticut, Storrs, CT \\ 06269, USA \\ ${ }^{3}$ Department of Physics and Engineering Physics, Tulane University, New Orleans, LA \\ 70118, USA \\ *mmontemore@tulane.edu
}




\begin{abstract}
Single-atom alloys can be effective catalysts and have been compared to supported single-atom catalysts. To rationally design single-atom alloys and other surfaces with localized ensembles, it is crucial to understand variations in reactivity when varying the dopant and the ensemble size. Here, we examined hydrogen adsorption on surfaces embedded with localized clusters and discovered general trends. Counterintuitively, increasing the amount of a more reactive metal sometimes makes a surface site less reactive. This behavior is due to the hybridization and splitting of sharp peaks in the electronic density of states of many of these surfaces, making them analogous to free-standing nanoclusters. Further, single-atom alloys have qualitatively different behavior than larger ensembles. Specifically, the adsorption energy is Ushaped when plotted against the dopant's group for single atom alloys. Additionally, adsorption energies on single atom alloys correlate more strongly with the dopant's p-band center than the d-band center.
\end{abstract}




\section{Introduction}

Rationalizing and designing catalysts requires a strong understanding of how active sites behave upon modification. For alloy catalysts, it is particularly crucial to understand how active site reactivity-often measured with an adsorption energy-changes upon modifying the composition of the active site. Intuitively, we expect that as the composition of a surface site is smoothly varied, the binding strength of species to the site will vary smoothly as well. Indeed, this has been shown to be the case in many systems. ${ }^{1-5}$ For example, density functional theory (DFT) calculations show that alloying increasing amounts of $\mathrm{Ag}$ or $\mathrm{Pd}$ into $\mathrm{Au}$ causes the adsorption strength of $\mathrm{O}$ to smoothly increase. Similarly, experiments of gas exposure to alloy single crystals often show smooth, monotonic changes in uptake as composition is changed.,.$^{2,6,7}$ While changes in composition could cause overall catalytic performance to change in a jagged or nonmonotonic fashion due to changes in a variety of factors (e.g., kinetics, surface segregation, reconstruction, particle size), and the reactivity of a site of a fixed composition can be influenced by its local environment, ${ }^{8}$ the affinity of a particular site for a given species generally changes smoothly as composition is varied.

Small, localized ensembles of dopant atoms embedded in the surface of a host have recently gained significant attention, as they can be effective catalysts for many reactions, such as selective hydrogenation..$^{9-11}$ Single-atom alloys have received particular attention, including development of models and correlations for predicting or understanding adsorption energies. ${ }^{12-14}$ Single-atom alloys can have unusual properties, such as allowing spillover and breaking linear correlations between energies. ${ }^{15-17}$ Further, they often feature localized, sharp electronic states..$^{13,18,19}$ As this is similar to the behavior of nanoclusters (small particles with only a few atoms), ${ }^{20}$ alloy surfaces with localized ensembles have been called "embedded nanoclusters". ${ }^{21}$ 
In this work, we show how localized clusters of atoms embedded in the surface of a host can defy intuition and link this counterintuitive behavior to hybridization and splitting of sharp dstate peaks in these systems. By studying many dopants and several hosts, we also elucidate trends in the behavior of these systems, which allows us to develop correlations between electronic structure and adsorption energies for single atom alloys and embedded nanoclusters. In particular, we show that single-atom alloys have qualitatively different behavior from dimers and trimers in how the $\mathrm{H}$ adsorption energy depends on the electronic structure.

\section{Methods}

Plane-wave DFT calculations were performed with the VASP code. ${ }^{22,23}$ The k-point mesh was $7 \times 7 \times 1$ with a $3 \times 3$ surface cell of 4 layers, with the bottom two layers fixed. The PBE exchange-correlation functional was used, with the Tkatchenko-Scheffler method ${ }^{24}$ accounting for van der Waals interactions. The electronic convergence criterion was $10^{-5} \mathrm{eV}$. Adsorbates were placed in the fcc hollow site and relaxed to a force tolerance of 0.03 to $0.035 \mathrm{eV} / \AA$. Plane wave cut-offs were set to $400 \mathrm{eV}$, and all calculations were spin-polarized. H adsorption energies were defined relative to gas-phase $\mathrm{H}_{2}$, with the convention that exothermic adsorption is negative. The calculated, pure-metal lattice constants were used, as doping a small, localized cluster into a surface does not change the lattice constant.

\section{Results and Discussion}

To elucidate trends in adsorption on embedded nanoclusters, we performed DFT calculations for H adsorption on late transition metal fcc(111) surfaces substitutionally doped with one, two, and three metal atoms. We focused on Ag as the host element, but also examined $\mathrm{Au}, \mathrm{Cu}$, and $\mathrm{Pd}$ as hosts for comparison, and we included 19 other d-block metals (Co, $\mathrm{Cr}, \mathrm{Fe}$, Hf, Ir, Mn, Mo, Ni, Pt, Re, Rh, Ru, Sc, Ta, Tc, Ti, V, W, Zn) as dopants. We denote $n$ dopants of 
a guest $\mathrm{X}$ dopant into a host $\mathrm{Y}$ as $\mathrm{X}_{\mathrm{n}} \mathrm{Y}$, such that $\mathrm{Cu}_{1} \mathrm{Ag}$ denotes a single $\mathrm{Cu}$ atom doped into a $\operatorname{Ag}(111)$ surface.

Doping a single heteroatom into a Ag surface nearly always strengthens $\mathrm{H}$ adsorption, as Ag binds $\mathrm{H}$ relatively weakly. $\mathrm{H}$ prefers to adsorb in hollow sites on pure metal surfaces, and hence we generally expect that adding more of the reactive metal to the adsorption site will further strengthen $\mathrm{H}$ adsorption, as it can now bond to more atoms of that metal. In other words, intuition suggests that the trends should be monotonic, with adsorption energies varying roughly linearly with the number of dopants added. This is indeed the case for $\mathrm{Cu}$ (Figure 1a): adding increasing amounts of $\mathrm{Cu}$ to the adsorption site stabilizes $\mathrm{H}$ approximately linearly, as $\mathrm{H}$ is able to bond to more $\mathrm{Cu}$ atoms and pure $\mathrm{Cu}$ adsorbs $\mathrm{H}$ more strongly than pure $\mathrm{Ag}$. However, for Mo, adding a second dopant atom significantly weakens $\mathrm{H}$ adsorption compared to the single-Mo case, to such a degree that $\mathrm{Mo}_{2} \mathrm{Ag}$ adsorbs $\mathrm{H}$ even weaker than pure $\mathrm{Ag}$. This is a significant departure from intuition, given that pure Mo adsorbs $\mathrm{H}$ much more strongly than pure $\mathrm{Ag}$. Similarly, adsorption on $\mathrm{W}_{\mathrm{n}} \mathrm{Ag}$ follows a monotonically decreasing trend when up to two dopants are added, but deviates from this trend when a third dopant atom is added.
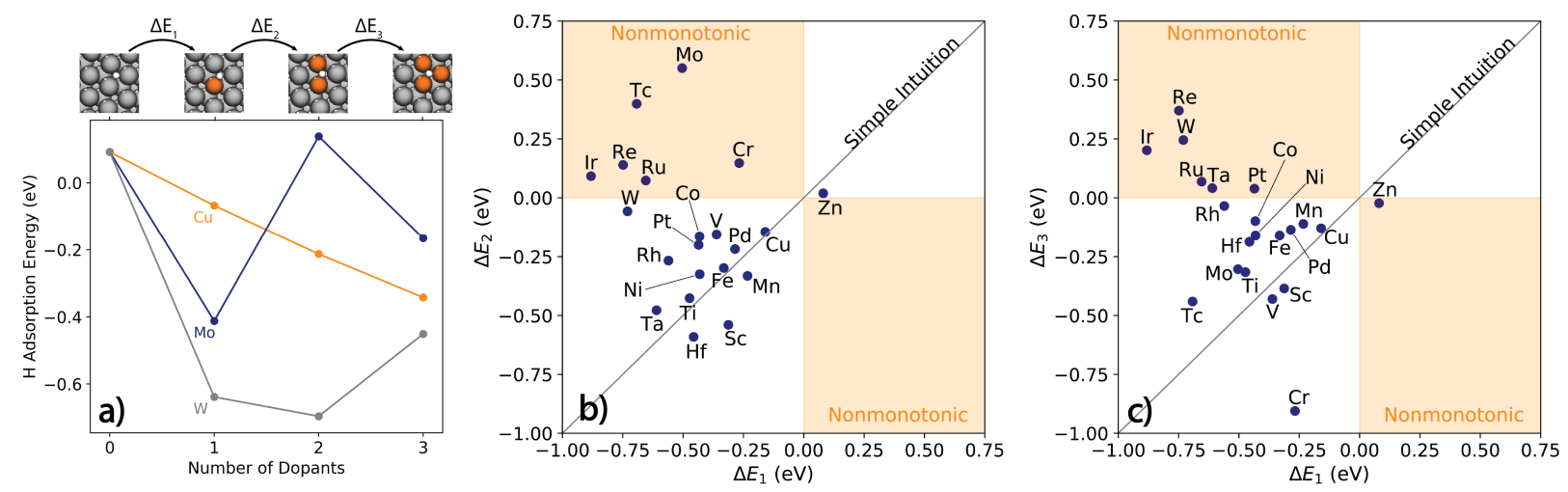

Figure 1. Nonmonotonic trends in adsorption for substitutional doping into $\operatorname{Ag}(111)$. a) Hydrogen adsorption energy vs. number of dopants. Graphics show relaxed geometries for $\mathrm{Cu}$ doped into Ag. b) The difference in $\mathrm{H}$ adsorption energy between pure Ag and a single dopant 
$\left(\Delta \mathrm{E}_{1}\right)$ vs. the difference in adsorption energy between one dopant and two dopants $\left(\Delta \mathrm{E}_{2}\right)$. c) $\Delta \mathrm{E}_{1}$ vs. the difference in adsorption energy between two dopants and three dopants $\left(\Delta \mathrm{E}_{3}\right)$. In parts $b$ and c, the "Simple Intuition" line shows the case where adding a second or third dopant has the same effect on the adsorption energy as adding the first dopant.

More generally, we can elucidate trends by examining how the $\mathrm{H}$ adsorption energy differs between surfaces with consecutive numbers of dopants. We defined $\Delta \mathrm{E}_{1}, \Delta \mathrm{E}_{2}$, and $\Delta \mathrm{E}_{3}$ as the change in adsorption energy upon a change from zero to one, one to two, and two to three dopants, respectively (Figure 1a). Based on simple intuition, we generally expect that $\Delta \mathrm{E}_{1}, \Delta \mathrm{E}_{2}$, and $\Delta \mathrm{E}_{3}$ should be roughly similar. ${ }^{1-5}$ This is indeed the case for many of the dopant elements. However, we found that several dopants feature the counterintuitive, nonmonotonic trends seen for Mo and W in Figure 1a. For example, for Mo, Ir, Ru, Cr, Tc, and Re, $\Delta \mathrm{E}_{1}$ and $\Delta \mathrm{E}_{2}$ are quite different, and in fact have different signs (Figure 1b), corresponding to unexpected weakening of adsorption upon adding the second dopant atom. For Ir, W, Ru, Ta, Re, and Pt, $\Delta \mathrm{E}_{1}$ and $\Delta \mathrm{E}_{3}$ have different signs (Figure 1c), corresponding to unexpected weakening of adsorption upon adding the third dopant. Whether a particular dopant features a nonmonotonic trend appears to have no correlation to the absolute adsorption energy. For example, $\mathrm{Mo}_{1} \mathrm{Ag}$ and $\mathrm{Ti}_{1} \mathrm{Ag}$ have similar adsorption energies ( $-0.41 \mathrm{eV}$ and $-0.38 \mathrm{eV}$, respectively), but $\mathrm{Mo}_{2} \mathrm{Ag}$ and $\mathrm{Ti}_{2} \mathrm{Ag}$ have adsorption energies that differ by nearly $1 \mathrm{eV}(+0.14 \mathrm{eV}$ and $-0.81 \mathrm{eV})$.

These nonmonotonic trends were also observed for some dopants embedded in other inert metal surfaces (Figure 2). A given dopant generally behaves fairly similarly when embedded in $\mathrm{Cu}, \mathrm{Ag}$, or $\mathrm{Au}$. For example, doping $\mathrm{Mo}$ and $\mathrm{Cr}$ into all of these hosts gives nonmonotonic behavior, while doping Pd gives nearly linear behavior in all three cases. As a host, Pd behaves somewhat differently than $\mathrm{Au}, \mathrm{Ag}$, and $\mathrm{Cu}$, partially because it adsorbs $\mathrm{H}$ more strongly than the coinage metals. However, nonmonotonic behavior is still observed in some cases, such as Mo 
and Co. In these cases, adding a single dopant atom weakens $\mathrm{H}$ adsorption, but adding a second dopant atom strengthens it.
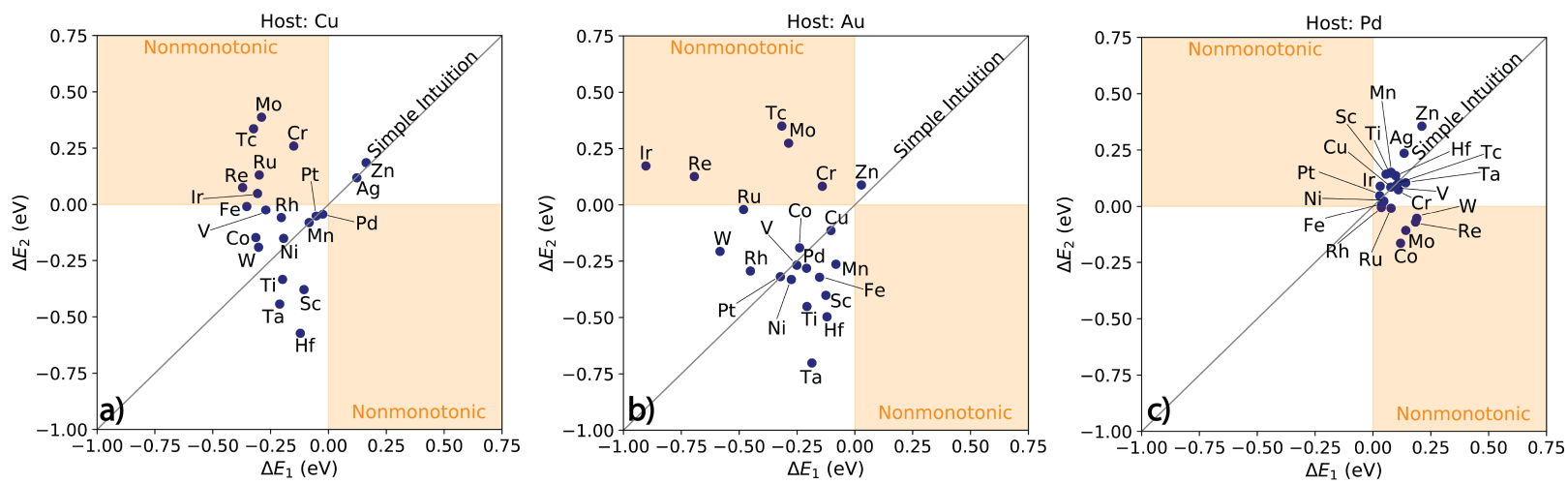

Figure 2. $\mathrm{H}$ adsorption trends for single atoms and dimers in various hosts. $\Delta \mathrm{E}_{2}$ vs. $\Delta \mathrm{E}_{1}$ for a) $\mathrm{Cu}, \mathrm{b}) \mathrm{Au}$, and c) $\mathrm{Pd}$.

To understand the source of the nonmonotonic behavior, we examined the projected density of states (PDOS) on the dopant atoms. Single-atom alloys often feature a single, sharp peak, somewhat similar to an electronic state in an atom in the gas-phase. ${ }^{13,18}$ We found that twoatom and three-atom ensembles also often have sharp d-state peaks, but due to dopant-dopant hybridization and splitting there are often two peaks for dimers and three peaks for trimers (Figure 3a,b). This is expected based on a molecular orbital theory picture of atom-like states. There are sometimes additional peaks due to hybridization among $\mathrm{d}, \mathrm{p}$, and s states, as well as hybridization with surrounding Ag atoms. 

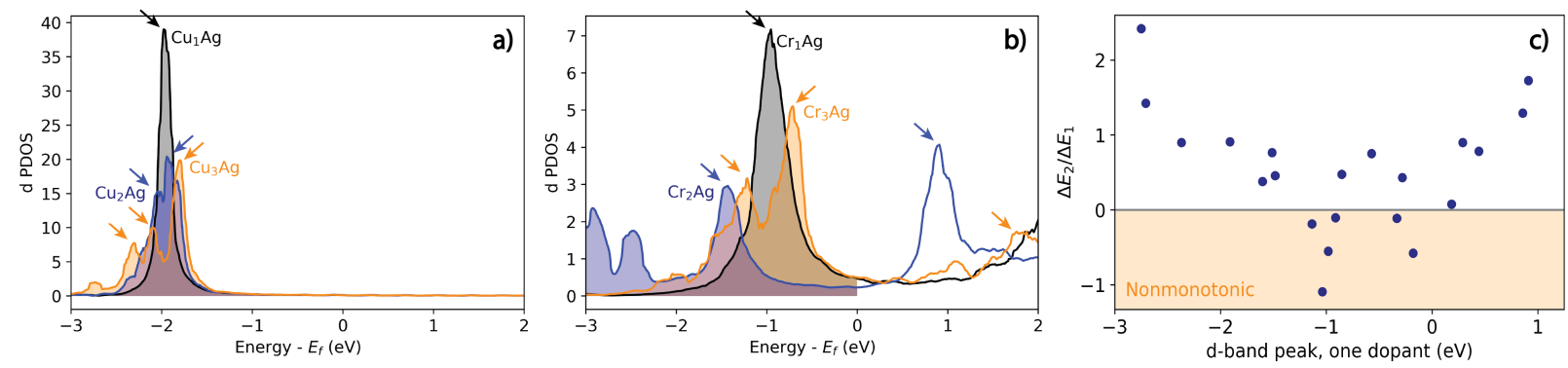

Figure 3. Nonmonotonic trends are due to splitting of localized d-states across the Fermi energy. $\mathrm{a}, \mathrm{b}) \mathrm{PDOS}$ for the d-states on $\mathrm{Cu}$ atoms in $\mathrm{Cu}_{\mathrm{n}} \mathrm{Ag}$ (a monotonic case) and $\mathrm{Cr}$ atoms in $\mathrm{Cr}_{\mathrm{n}} \mathrm{Ag}$ (a nonmonotonic case). The most relevant peaks are indicated with arrows. c) $\Delta \mathrm{E}_{2} / \Delta \mathrm{E}_{1}$ vs. the $\mathrm{d}-$ band peak for the single-atom alloys, relative to the Fermi energy.

Examination of the PDOS indicates that the splitting of these localized $d$ states causes the nonmonotonic trends in adsorption energies. For the two-dopant cases, the d-state peaks can be split across the Fermi energy such that the upper peak becomes unoccupied and the lower peak has a low energy, as seen for $\mathrm{Cr}_{2} \mathrm{Ag}$ (Figure 3b). This results in a low d-band center and a low number of states near the Fermi energy, both of which lead to weak adsorption. ${ }^{25-27}$ This splitting across the Fermi energy often occurs when the single-atom alloy's d-band peak is less than $\sim 1 \mathrm{eV}$ below the Fermi energy, such that nonmonotonic behavior is often observed in these cases (Figure 3c). The precise amount of splitting and its precise effect on adsorption depends on the coupling and hybridization between dopant atoms and the hybridization between the dopant and the host metal atoms. This results in some variation in some scatter in Figure $3 \mathrm{c}$. We also found that $\Delta \mathrm{E}_{2} / \Delta \mathrm{E}_{1}$ correlates with the difference in d-band center between the one-dopant and twodopant cases, and that it has a similar trend as Figure $3 \mathrm{c}$ when plotted against the d-band center instead of the d-band peak (see Supporting Information). Hence, in embedded nanoclusters, hybridization and splitting of the localized d-state peaks determines trends across the number of 
dopants. Nonmonotonic trends in adsorption energies occur when splitting drives states significantly above and below the Fermi energy.

The link between electronic structure and the nonmonotonic trends suggests that localized clusters will behave more counterintuitively than more "bulk-like" alloys. To test this, we calculated $\mathrm{H}$ adsorption energies on bulk-like MoAg and IrAg alloys in hollow sites with one or two dopant atoms. These calculations indeed show that the bulk-like alloys have behavior closer to intuition than the embedded nanoclusters (Figure S3). Hence, we generally expect the nonmonotonic behavior to be quite rare and much less significant for bulk-like alloys, due to the lack of sharp peaks in their DOS.

While the d-band peaks and splitting explain trends across the number of dopant atoms, it is also important to understand trends across the type of dopant. For a given host, we found that the $\mathrm{H}$ adsorption energy is linked to the dopant's group in the periodic table (Figure 4). Dopants in the same periodic group often have similar adsorption energies, and this relationship is particularly distinct for single-atom alloys. This is consistent with previous work suggesting that the idealized d-band filling is important in adsorption on transition metal alloys ${ }^{25,28,29}$ and recent work on Cu-based single-atom alloys that found smooth relationships between the dopant's group and the adsorption energy of methane, although with a different trend. ${ }^{30}$

For $\mathrm{Ag}$-host and $\mathrm{Cu}$-host surfaces, the exceptions to this similarity within a dopant group for single-atom alloys are nearly always cases where the surface has a magnetic moment. Most cases that follow the primary, $\mathrm{U}$-shaped relationship have a magnetic moment near 0 , while the outliers have significant magnetic moments (Figure S1). For single-atom alloys with Au as the host, there is a systematic variation in adsorption energies within each group for groups 6 
through 9. In this case, there is a smooth relationship for each period, with the $\mathrm{H}$ binding generally stronger as $5 \mathrm{~d}$ metals $>4 \mathrm{~d}$ metals $>3 \mathrm{~d}$ metals (Figure S2). This increased variation within each group is likely due to Au's more extended d-orbitals, which may lead to higher hybridization with the dopant's orbitals and hence more variation among dopants of the same group.

The relationship between the $\mathrm{H}$ adsorption energy and the dopant's group is U-shaped for $\mathrm{Cu}$-host, Ag-host, and Au-host single-atom alloy surfaces (Figure 4a-d). This is significantly different behavior from pure metal surfaces, where $\mathrm{H}$ adsorption is stronger on early transition metals. For example, $\mathrm{Rh}_{1} \mathrm{Ag}$ adorbs $\mathrm{H}$ somewhat more strongly than $\mathrm{Ti}_{1} \mathrm{Ag}(-0.47$ and $-0.38 \mathrm{eV}$, respectively), while our previous calculations ${ }^{25}$ show the opposite trend on pure surfaces: pure Ti adsorbs $\mathrm{H}$ more strongly than pure $\mathrm{Rh}$ by $0.46 \mathrm{eV}$. As the number of dopants increases, the adsorption energy vs. dopant group curve changes shape, such that dopants from the left side of the d-block adsorb $\mathrm{H}$ more strongly than those from the right side (Figure $4 \mathrm{e}-\mathrm{j}$ ). This is closer to the expected trend for pure metal surfaces. As the number of dopant atoms increases, the more the dopant peaks split and broaden, leading to more wider bands, more hybridization with the host, and more similar behavior to pure metals or bulk alloys. We also studied these trends for a Ag surface with one dopant in the surface layer and one in the subsurface layer (Figure $4 \mathrm{j}$ ). In this case, there is still a U-shaped curve, but there is increased variation within a dopant group, likely due to dopant-dopant hybridization. For Pd-host surfaces, the adsorption energy curve is fairly flat and slightly S-shaped, with a similar shape for one and two dopants. 


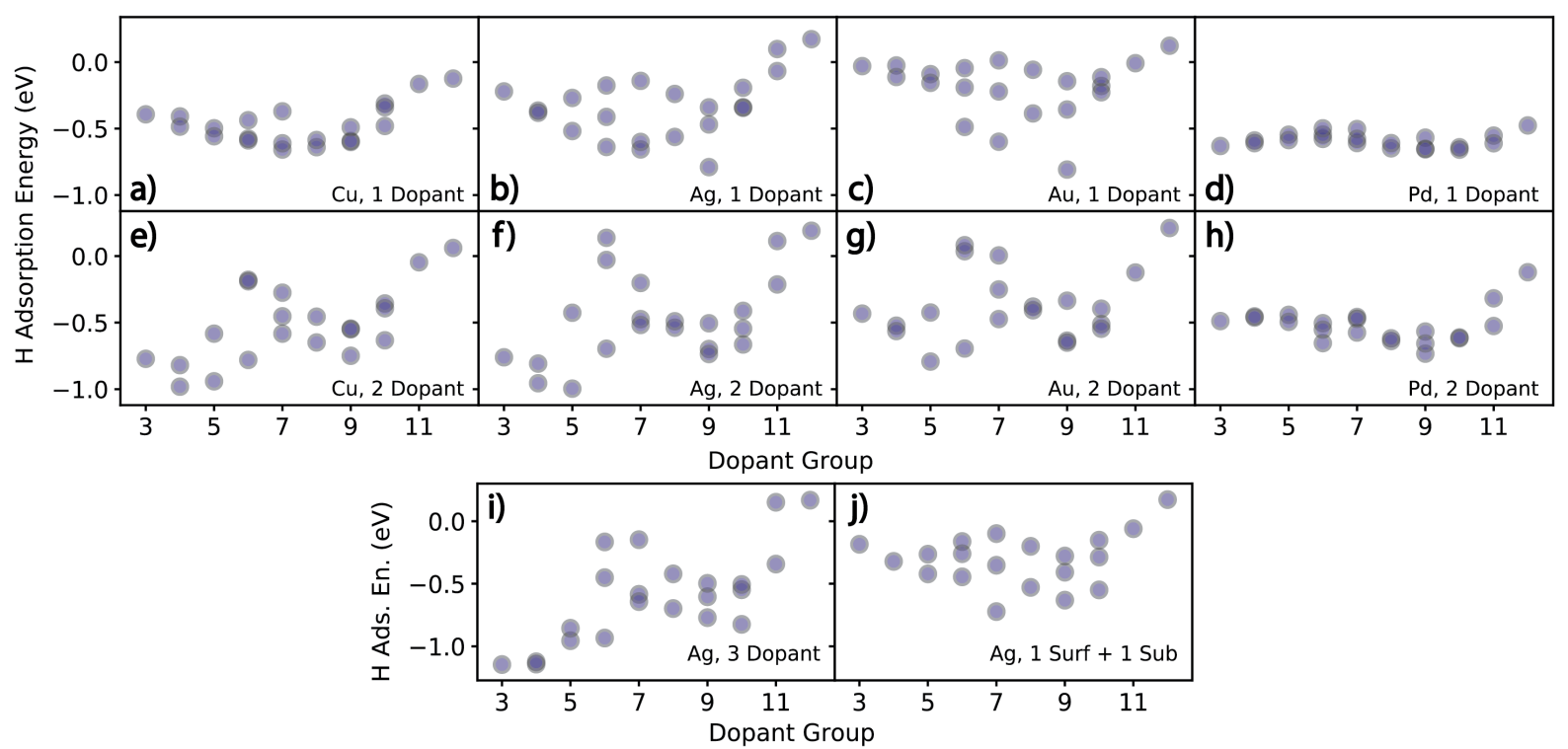

Figure 4. $\mathrm{H}$ adsorption energies a function of dopant group in each host metal for a-d) 1 dopant surfaces; e-h) 2 dopant surfaces; i) 3 dopant surfaces; j) surfaces with a dopant in the surface and a neighboring dopant in the second layer.

In addition to the change in the overall shape of the curve, the relationship between the adsorption energy and dopant group also becomes less distinct in surfaces with multiple dopants than for single-atom alloys (e.g., Figure 4e vs. Figure 4a). Again, this increased variation within groups is likely due to increased hybridization between the dopant atoms and their neighbors in the larger ensembles, resulting in a more bulk-like PDOS and more variation among metals in the same group.

Links between adsorption energies and electronic structure properties provide key insights into changes in reactivity. To understand how the electronic structure of the surface affects the adsorption strength, we calculated band properties from the PDOS on the dopant atoms for the Ag-host bare surfaces with one, two, or three dopants. Broadly, these calculations suggest that the behavior of these systems is controlled by properties of the dopant's p-band and 
d-band, as suggested by previous work on transition metal alloy surfaces ${ }^{25,28,29}$ However, the importance of these two bands changes for different numbers of dopants.

For single-atom alloys with a Ag host, variations in the adsorption energy are primarily controlled by the dopant's p band (Figure 5a,d). The p-band center correlates well with the adsorption energy $\left(R^{2}=0.73\right)$, while the d-band center correlates much less strongly with the adsorption energy $\left(R^{2}=0.37\right)$. Most of the correlation for the d-band center comes from the two points with the lowest d-band centers, $\mathrm{Au}_{1} \mathrm{Ag}$ and $\mathrm{Mn}_{1} \mathrm{Ag}$. Indeed, removing $\mathrm{Au}$ reduces the correlation for the d-band center on single-atom alloys to $R^{2}=0.16 . \mathrm{Zn}_{\mathrm{n}} \mathrm{Ag}$ and $\mathrm{Tc}_{\mathrm{n}} \mathrm{Ag}$ were removed from the d-band center plots because they are outliers, but including them does not change the qualitative trends (Figure S6). As the number of dopants increases to two or three, the p-band center correlates less strongly with the adsorption energy, but the d-band center correlates more strongly. Further, the correlation between the adsorption energy and the p-band center is positive for single-atom alloys, but is negative for two or three dopants. Hence, adsorption on single-atom alloys has a qualitatively different dependence on the dopants' electronic structure than adsorption on larger ensembles. This change may be due to differences in the p-band DOS: for single-atom alloys, the $\mathrm{p}$ band generally has two broad peaks, while for larger ensembles the $\mathrm{p}$ band has many sharper peaks (Figure S8). The relationship between the p-band center and the adsorption energy for single-atom alloys also explains the $\mathrm{U}$ shape for the adsorption energy vs. group curve, as the p-band center has a U-shaped dependence on dopant group for single-atom alloys but decreases from left to right for two and three atom ensembles (Figure S7). Overall, the d-band model generally assumes a broad, flat sp-band and that the d-band shape does not change too dramatically between surfaces,${ }^{27}$ and it is therefore reasonable that it may partially break down or behave differently for single-atom alloys. 


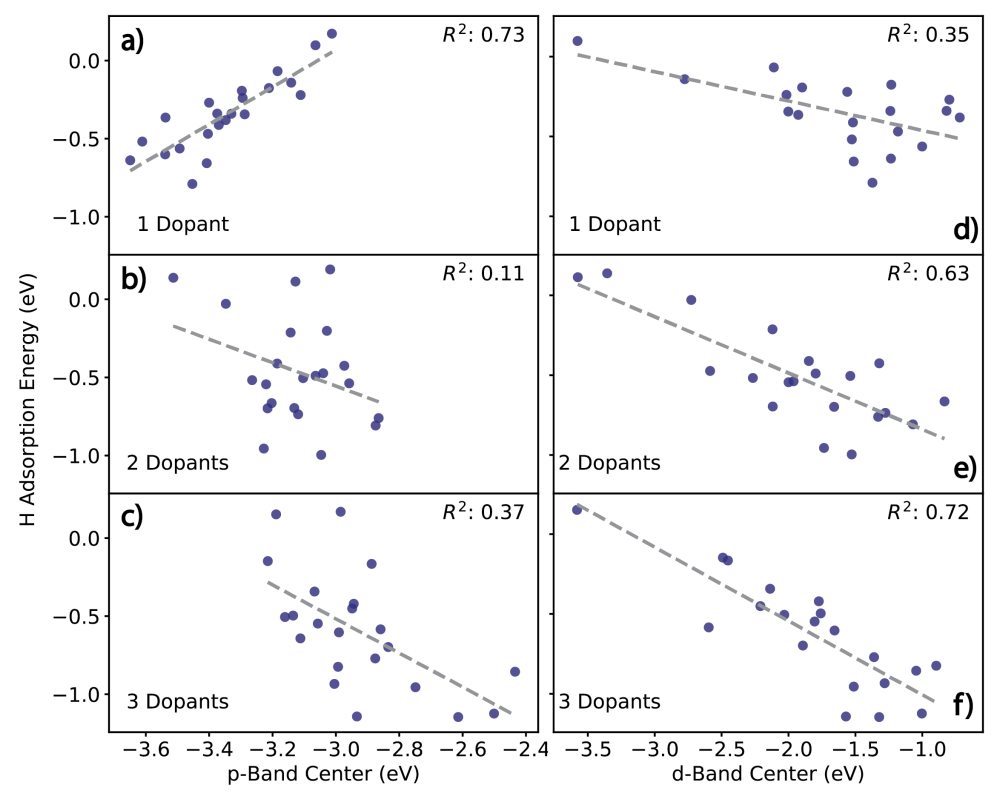

Figure 5. $\mathrm{H}$ adsorption energies on $\mathrm{Ag}$ surfaces doped with various metals as a function of the dopant atoms' a) p-band center, and b) d-band center. Linear fits and corresponding $\mathrm{R}^{2}$ values are shown. The dopants' p-band controls adsorption for single-atom alloys, while the d-band controls adsorption for the three-dopant case.

Overall, the magnetic behavior of these embedded nanocluster systems can be somewhat complicated. In most cases that have a nonzero magnetic moment, adsorbed $\mathrm{H}$ somewhat reduces the magnetic moment relative to the bare surface (Figure S4), as seen in previous work on magnetic nanoclusters. ${ }^{31}$ In a few cases, such as $\mathrm{Mn}_{3} \mathrm{Ag}, \mathrm{H}$ has a very large effect on the magnetic moment. For Pd-host materials, the dopant appears to induce magnetism in surrounding Pd atoms in many cases, similar to what has been observed in previous work on impurities in Pd. ${ }^{32}$ Further discussion is in the Supporting Information.

We also performed a few test calculations to compare $\mathrm{H}$ adsorption to $\mathrm{C}$ and $\mathrm{O}$ adsorption. These calculations suggest that trends for $\mathrm{C}$ and $\mathrm{O}$ across various dopants are similar to those for $\mathrm{H}$ (Figure S9). For example, $\mathrm{Cu}$ doped into $\mathrm{Ag}$ gives monotonic trends in adsorption 
energies for all three adsorbates, while Mo doped into Ag gives nonmonotonic trends for all three adsorbates.

\section{Conclusion}

We elucidate trends in adsorption on embedded nanoclusters, both across number of dopant atoms and across dopants. We find that the unusual electronic structure of embedded nanoclusters, and especially single-atom alloys, leads to different behavior from other types of alloy surfaces. Counterintuitive nonmonotonic trends upon increasing the number of dopants are due to splitting of free-atom-like electronic states. When the single-atom d-state peak is within $\sim 1 \mathrm{eV}$ of the Fermi energy, the two d-state peaks in the dimer may be split such that one is above the Fermi energy and one is significantly below the Fermi energy, leading to weak adsorption. The significant changes in adsorption energy are consistent with the well-known feature of supported or unsupported nanoclusters that properties can change significantly by adding a single atom. We found that single-atom alloys display unusual behavior, even compared to larger embedded nanoclusters. Specifically, trends across the d-block are different, as the adsorption energy vs. dopant group curve is U-shaped for single-atom alloys instead of generally increasing from left to right as is the case for larger ensembles and pure metals. Single-atom alloys also have a different dependence on electronic structure: the dopant atoms' p-band controls variations in adsorption energy more strongly than the d-band, while the d-band has a larger impact for larger ensembles and pure metals. Further, the relationship between the adsorption energy and the $\mathrm{p}$-band center is positive for single-atom alloys but negative for two and three atom ensembles. 


\section{Supporting Information}

See the supporting information for discussion of bulk-like alloys, magnetic effects, further methods, electronic structure effects and trends, and $\mathrm{C}$ and $\mathrm{O}$ adsorption energies.

\section{Acknowledgments}

We gratefully acknowledge support from Tulane University. A.M. acknowledges support from the National Science Foundation from grant DMR-1852274, and M.M.M. acknowledges support from the Louisiana Board of Regents Research Competitiveness Subprogram, Contract Number LEQSF(2019-22)-RD-A-20. This research was supported in part using computing resources provided by Technology Services at Tulane University, New Orleans, LA, and computational resources provided by the Louisiana Optical Network Infrastructure (http://www.loni.org). We acknowledge useful discussions with E. C. H. Sykes.

\section{References}

${ }^{1}$ M.M. Montemore, R.J. Madix, and E. Kaxiras, J. Phys. Chem. C 120, 16636 (2016).

${ }^{2}$ W.-Y. Yu, L. Zhang, G.M. Mullen, G. Henkelman, and C.B. Mullins, J. Phys. Chem. C 119, 11754 (2015).

${ }^{3}$ H. Li, K. Shin, and G. Henkelman, J. Chem. Phys. 149, 174705 (2018).

${ }^{4}$ N. Takehiro, P. Liu, A. Bergbreiter, J.K. Nørskov, and R.J. Behm, Phys. Chem. Chem. Phys. 16, 23930 (2014).

${ }^{5}$ Y.M. Choi, C. Compson, M.C. Lin, and M. Liu, J. Alloys Compd. 427, 25 (2007).

${ }^{6}$ M.M. Montemore, E.D. Cubuk, J.E.E. Klobas, M. Schmid, R.J. Madix, C.M. Friend, and E. 
Kaxiras, Phys. Chem. Chem. Phys. 18, 26844 (2016).

${ }^{7}$ C.C. Leon, J.G. Lee, and S.T. Ceyer, J. Phys. Chem. C 118, 29043 (2014).

${ }^{8}$ M.T. Darby, E.C.H. Sykes, A. Michaelides, and M. Stamatakis, Top. Catal. 61, 428 (2018).

${ }^{9}$ J. Liu, M.B. Uhlman, M.M. Montemore, A. Trimpalis, G. Giannakakis, J. Shan, S. Cao, R.T. Hannagan, E.C.H. Sykes, and M. Flytzani-Stephanopoulos, ACS Catal. 9, 8757 (2019).

${ }^{10}$ G. Kyriakou, M.B. Boucher, A.D. Jewell, E.A. Lewis, T.J. Lawton, A.E. Baber, H.L. Tierney, M. Flytzani-Stephanopoulos, and E.C.H. Sykes, Science 335, 1209 (2012).

${ }^{11}$ G. Giannakakis, M. Flytzani-Stephanopoulos, and E.C.H. Sykes, Acc. Chem. Res. 52, 237 (2019).

${ }^{12}$ S. Saxena, T.S. Khan, F. Jalid, M. Ramteke, and M.A. Haider, J. Mater. Chem. A (2019).

${ }^{13}$ M.T. Greiner, T.E. Jones, S. Beeg, L. Zwiener, M. Scherzer, F. Girgsdies, S. Piccinin, M. Armbrüster, A. Knop-Gericke, and R. Schlögl, Nat. Chem. 10, 1008 (2018).

${ }^{14}$ A. Dasgupta, Y. Gao, S.R. Broderick, E.B. Pitman, and K. Rajan, J. Phys. Chem. C 124, 14158 (2020).

${ }^{15}$ M.T. Darby, M. Stamatakis, A. Michaelides, and E.C.H. Sykes, J. Phys. Chem. Lett. 9, 5636 (2018).

${ }^{16}$ M.M. Montemore and J.W. Medlin, J. Phys. Chem. C 117, 20078 (2013).

${ }^{17}$ G. Sun, Z.-J. Zhao, R. Mu, S. Zha, L. Li, S. Chen, K. Zang, J. Luo, Z. Li, S.C. Purdy, A.J. Kropf, J.T. Miller, L. Zeng, and J. Gong, Nat. Commun. 9, 4454 (2018). 
${ }^{18}$ H. Thirumalai and J.R. Kitchin, Top. Catal. 61, 462 (2018).

${ }^{19}$ V. Fung, G. Hu, and B. Sumpter, J. Mater. Chem. A 8, 6057 (2020).

${ }^{20}$ R. Jin, C. Zeng, M. Zhou, and Y. Chen, Chem. Rev. 116, 10346 (2016).

${ }^{21}$ R.A. Hoyt, M.M. Montemore, I. Fampiou, W. Chen, G. Tritsaris, and E. Kaxiras, J. Chem. Inf. Model. 59, 1357 (2019).

${ }^{22}$ G. Kresse and J. Furthmüller, Comput. Mater. Sci. 6, 15 (1996).

${ }^{23}$ G. Kresse and J. Hafner, Phys. Rev. B 47, 558 (1993).

${ }^{24}$ A. Tkatchenko and M. Scheffler, Phys. Rev. Lett. 102, 073005 (2009).

${ }^{25}$ M.M. Montemore and J.W. Medlin, J. Am. Chem. Soc. 136, 9272 (2014).

${ }^{26}$ M. Rangan, M.M. Yung, and J.W. Medlin, Catal. Letters 142, 718 (2012).

${ }^{27}$ B. Hammer and J.K. Nørskov, in Adv. Catal. (Elsevier, 2000), pp. 71-129.

${ }^{28}$ M.M. Montemore and J.W. Medlin, J. Phys. Chem. C 118, 2666 (2014).

${ }^{29}$ M.M. Montemore, C.F. Nwaokorie, and G.O. Kayode, Catal. Sci. Technol. 10, 4467 (2020).

${ }^{30}$ V. Fung, G. Hu, and B. Sumpter, J. Mater. Chem. A 8, 6057 (2020).

${ }^{31}$ R.A. Hoyt, M.M. Montemore, and E. Kaxiras, J. Phys. Chem. Lett. 9, 5339 (2018).

${ }^{32}$ A. Oswald, R. Zeller, and P.H. Dederichs, J. Magn. Magn. Mater. 54-57, 969 (1986). 